\title{
ON THE AVERAGE OF THE NUMBER OF IMAGINARY QUADRATIC FIELDS WITH A GIVEN CLASS NUMBER
}

\author{
YOUNESS LAMZOURI
}

\begin{abstract}
Let $\mathcal{F}(h)$ be the number of imaginary quadratic fields with class number $h$. In this note, we improve the error term in Soundararajan's asymptotic formula for the average of $\mathcal{F}(h)$. Our argument leads to a similar refinement of the asymptotic for the average of $\mathcal{F}(h)$ over odd $h$, which was recently obtained by Holmin, Jones, Kurlberg, McLeman and Petersen.
\end{abstract}

\section{INTRODUCTION}

An important problem in number theory, which goes back to Gauss, is to determine all imaginary quadratic fields with a given class number. Let $\mathcal{F}(h)$ be the number of imaginary quadratic fields with class number $h$. Then for instance one has $\mathcal{F}(1)=$ 9, which follows from the celebrated solution of Baker-Stark-Heegner to Gauss' class number 1 problem for imaginary quadratic fields. In [3], Soundararajan studied the quantity $\mathcal{F}(h)$ and determined its average order. More precisely, he proved that for any $\epsilon>0$

$$
\sum_{h \leq H} \mathcal{F}(h)=\frac{3 \zeta(2)}{\zeta(3)} H^{2}+O_{\epsilon}\left(\frac{H^{2}}{(\log H)^{1 / 2-\epsilon}}\right) .
$$

The purpose of this note is to improve the error term in this asymptotic formula.

Theorem 1.1. We have

$$
\sum_{h \leq H} \mathcal{F}(h)=\frac{3 \zeta(2)}{\zeta(3)} H^{2}+O\left(\frac{H^{2}(\log \log H)^{3}}{\log H}\right) .
$$

In a recent work [2], Holmin, Jones, Kurlberg, Mcleman and Petersen studied statistics of the class numbers of imaginary quadratic fields. In particular, they used the Cohen-Lenstra heuristics together with the work of Granville and Soundararajan [1] on the distribution of values of $L\left(1, \chi_{d}\right)$ to formulate a conjecture on the asymptotic nature of $\mathcal{F}(h)$ as $h \rightarrow \infty$ through odd values. They also obtained the analogue of (1.1) for the average of $\mathcal{F}(h)$ over odd values of $h$, conditionally on the generalized Riemann

2010 Mathematics Subject Classification. Primary 11R29; Secondary 11R11, 11M20.

The author is partially supported by a Discovery Grant from the Natural Sciences and Engineering Research Council of Canada. 
hypothesis GRH. More precisely, they showed that assuming GRH

$$
\sum_{\substack{h \leq H \\ h \text { odd }}} \mathcal{F}(h)=\frac{15}{4} \frac{H^{2}}{\log H}+O_{\epsilon}\left(\frac{H^{2}}{(\log H)^{3 / 2-\epsilon}}\right) .
$$

Unlike (1.1) which is unconditional, the proof of (1.2) uses GRH to bound a certain character sum over primes, which appears in this case due to the fact that when $d>8$, the class number of $\mathbb{Q}(\sqrt{-d})$ is odd precisely when $d$ is prime, by genus theory.

The same argument in our proof of Theorem 1.1 leads to the following refinement of the asymptotic formula (1.2).

Theorem 1.2. Assume GRH. Then

$$
\sum_{\substack{h \leq H \\ h \text { odd }}} \mathcal{F}(h)=\frac{15}{4} \frac{H^{2}}{\log H}+O\left(\frac{H^{2}(\log \log H)^{3}}{(\log H)^{2}}\right) .
$$

The main ingredients in the proofs of (1.1) and (1.2) are asymptotic formulas for the complex moments of the class number $h(d)$ in a large uniform range (see (2.4) and (2.10) below). Using this approach, the best saving one can hope for in the error terms of Theorems 1.1 and 1.2 will be $1 / L$, if one can obtain an asymptotic formula for the average of $h(d)^{s}$, uniformly in $s$ such that $|s| \leq L$. It is also known (see [1]) that the current methods for computing these moments fail when $L \geq(\log H)(\log \log H)^{2}$. This shows that the saving of $(\log H) /(\log \log H)^{3}$ in the error terms of Theorems 1.1 and 1.2 constitute (up to the power of $\log \log H$ ) the limit of Soundararajan's method [3]. In particular, it would be interesting to improve the power of $\log H$ in the error terms of these results.

\section{Proofs of Theorems 1.1 and 1.2}

Let $X:=H^{2} \log \log H$. As in [3], it follows from Theorem 4 of [1] (concerning the distribution of extreme values of $L\left(1, \chi_{d}\right)$ ) together with Tatuzawa's refinement of the Landau-Siegel Theorem [4] that

$$
\sum_{h \leq H} \mathcal{F}(h)=\sum_{\substack{d \leq X \\ h(-d) \leq H}}^{b} 1+O_{A}\left(\frac{H^{2}}{(\log H)^{A}}\right)
$$

for any $A>0$, where $b$ indicates that the sum is over fundamental discriminants $-d$.

To estimate the main term in (2.1), Soundararajan used the following variant of Perron's formula

$$
\frac{1}{2 \pi i} \int_{c-i \infty}^{c+i \infty} \frac{x^{s}}{s}\left(\frac{(1+\delta)^{s+1}-1}{\delta(s+1)}\right) d s= \begin{cases}1 & \text { if } x \geq 1 \\ (1+\delta-1 / x) / \delta & \text { if }(1+\delta)^{-1} \leq x \leq 1 \\ 0 & \text { if } 0<x \leq(1+\delta)^{-1}\end{cases}
$$


Our improvement comes from using a different smooth cut-off function, namely

$$
I_{c, \lambda, N}(y):=\frac{1}{2 \pi i} \int_{c-i \infty}^{c+i \infty} y^{s}\left(\frac{e^{\lambda s}-1}{\lambda s}\right)^{N} \frac{d s}{s}
$$

where $c, \lambda>0$ are real numbers and $N$ is a positive integer. We prove

Lemma 2.1. Let $\lambda, c>0$ be real numbers and $N$ be a positive integer. Then we have

$$
I_{c, \lambda, N}(y) \begin{cases}=1 & \text { if } y>1 \\ \in[0,1] & \text { if } e^{-\lambda N} \leq y \leq 1 \\ =0 & \text { if } 0<y<e^{-\lambda N}\end{cases}
$$

Proof. First, we recall Perron's formula

$$
\frac{1}{2 \pi i} \int_{c-i \infty}^{c+i \infty} y^{s} \frac{d s}{s}= \begin{cases}1 & \text { if } y>1 \\ \frac{1}{2} & \text { if } y=1 \\ 0 & \text { if } 0<y<1\end{cases}
$$

Then, we observe that

$$
\frac{1}{2 \pi i} \int_{c-i \infty}^{c+i \infty} y^{s}\left(\frac{e^{\lambda s}-1}{\lambda s}\right)^{N} \frac{d s}{s}=\frac{1}{\lambda^{N}} \int_{0}^{\lambda} \cdots \int_{0}^{\lambda} \frac{1}{2 \pi i} \int_{c-i \infty}^{c+i \infty}\left(y e^{t_{1}+\cdots+t_{N}}\right)^{s} \frac{d s}{s} d t_{1} \cdots d t_{N} .
$$

By (2.2) $), \frac{1}{2 \pi i} \int_{c-i \infty}^{c+i \infty}\left(y e^{t_{1}+\cdots+t_{N}}\right)^{s} \frac{d s}{s} \in[0,1]$ for all values of $t_{i}$, and hence $I_{c, \lambda, N}(y) \in[0,1]$ for all $y>0$. The lemma follows from (2.2) upon noting that $y e^{t_{1}+\cdots+t_{N}}>1$ for all $t_{i} \in[0, \lambda]$ if $y>1$, and $y e^{t_{1}+\cdots+t_{N}}<1$ for all $t_{i} \in[0, \lambda]$ if $0<y<e^{-\lambda N}$.

Proof of Theorem 1.1. Let $c=1 / \log H, N$ be a positive integer, and $0<\lambda \leq 1$ be a real number to be chosen later. By (2.1) and Lemma 2.1 we obtain

$$
\sum_{h \leq H} \mathcal{F}(h) \leq \frac{1}{2 \pi i} \int_{c-i \infty}^{c+i \infty} \sum_{d \leq X}^{b} \frac{H^{s}}{h(-d)^{s}}\left(\frac{e^{\lambda s}-1}{\lambda s}\right)^{N} \frac{d s}{s}+O_{A}\left(\frac{H^{2}}{(\log H)^{A}}\right) \leq \sum_{h \leq e^{\lambda N} H} \mathcal{F}(h) .
$$

Let $T:=\log X /\left(10^{4}(\log \log X)^{2}\right)$. Then, it follows from equation (5) of [3] that

$$
\sum_{d \leq X}^{b} h(-d)^{-s}=3 \pi^{s-2} \cdot \mathbb{E}\left(L(1, \mathbb{X})^{-s}\right) \int_{1}^{X} x^{-s / 2} d x+O\left(X \exp \left(-\frac{\log X}{5 \log \log X}\right)\right),
$$

for all complex numbers $s$ with $\operatorname{Re}(s)=c$ and $|s| \leq T$, where

$$
L(1, \mathbb{X})=\prod_{p}\left(1-\frac{\mathbb{X}(p)}{p}\right)^{-1}
$$

and $\{\mathbb{X}(p)\}$ is a sequence of independent random variables taking the value 1 with probability $p /(2(p+1))$, 0 with probability $1 /(p+1)$, and -1 with probability $p /(2(p+$ $1)$ ). Note that $\mathbb{E}(\mathbb{X}(p))=0$ and $\mathbb{E}\left(\mathbb{X}(p)^{2}\right) \leq 1$, and hence the random product (2.5) converges almost surely, by Kolmogorov's three series theorems. 
Since $\left|e^{\lambda s}-1\right| \leq 3$ (if $H$ is large enough) and $h(-d) \geq 1$, it follows that the contribution of the region $|s|>T$ to the integral in (2.3) is

$$
\ll X\left(\frac{3}{\lambda}\right)^{N} \int_{\substack{|s|>T \\ \operatorname{Re}(s)=c}} \frac{|d s|}{|s|^{N+1}} \ll \frac{X}{N}\left(\frac{3}{\lambda T}\right)^{N} .
$$

Moreover, note that $\left|\left(e^{\lambda s}-1\right) / \lambda s\right| \leq 3$, if $H$ is large enough. Therefore, if follows from (2.4) that the integral in (2.3) equals

$$
\frac{1}{2 \pi i} \int_{\substack{|s| \leq T \\ \operatorname{Re}(s)=c}} \frac{3}{\pi^{2}} \cdot \mathbb{E}\left(L(1, \mathbb{X})^{-s}\right)\left(\int_{1}^{X} x^{-s / 2} d x\right)(\pi H)^{s}\left(\frac{e^{\lambda s}-1}{\lambda s}\right)^{N} \frac{d s}{s}+\mathcal{E}
$$

where

$$
\mathcal{E} \ll \frac{X}{N}\left(\frac{3}{\lambda T}\right)^{N}+\frac{3^{N} T}{c} X \exp \left(-\frac{\log X}{5 \log \log X}\right) .
$$

Choosing $\lambda=10 / T$ and $N=[A \log \log H]$, where $A>1$ is a constant, implies that

$$
\mathcal{E} \ll_{A} \frac{H^{2}}{(\log H)^{A}} .
$$

Furthermore, extending the main term of (2.6) to $\int_{c-i \infty}^{c+i \infty}$ shows that this integral equals

$$
\begin{aligned}
& \frac{1}{2 \pi i} \int_{c-i \infty}^{c+i \infty} \frac{3}{\pi^{2}} \cdot \mathbb{E}\left(L(1, \mathbb{X})^{-s}\right)\left(\int_{1}^{X} x^{-s / 2} d x\right)(\pi H)^{s}\left(\frac{e^{\lambda s}-1}{\lambda s}\right)^{N} \frac{d s}{s} \\
& +O_{A}\left(\mathbb{E}\left(L(1, \mathbb{X})^{-c}\right) \frac{X}{N}\left(\frac{3}{\lambda T}\right)^{N}\right) \\
= & \frac{3}{\pi^{2}} \cdot \mathbb{E}\left(\int_{1}^{X} I_{c, \lambda, N}\left(\frac{\pi H}{\sqrt{x}} L(1, \mathbb{X})^{-1}\right) d x\right)+O_{A}\left(\frac{H^{2}}{(\log H)^{A}}\right) .
\end{aligned}
$$

Now, it follows from Lemma 2.1 that for any $1 \leq x \leq X$ we have

$$
I_{c, \lambda, N}\left(\frac{\pi H}{\sqrt{x}} L(1, \mathbb{X})^{-1}\right) \begin{cases}=1 & \text { if } \sqrt{x} L(1, \mathbb{X}) \leq \pi H, \\ \in[0,1] & \text { if } \pi H<\sqrt{x} L(1, \mathbb{X}) \leq e^{\lambda N} \pi H, \\ =0 & \text { if } \sqrt{x} L(1, \mathbb{X})>\pi H e^{\lambda N}\end{cases}
$$

Thus we obtain

$$
\begin{aligned}
\mathbb{E}\left(\int_{1}^{X} I_{c, \lambda, N}\left(\frac{\pi H}{\sqrt{x}} L(1, \mathbb{X})^{-1}\right) d x\right) & =\mathbb{E}\left(\min \left(\frac{\pi^{2} H^{2}}{L(1, \mathbb{X})^{2}}, X\right)+O\left(\frac{H^{2}\left(e^{2 \lambda N}-1\right)}{L(1, \mathbb{X})^{2}}\right)\right) \\
& =\mathbb{E}\left(\min \left(\frac{\pi^{2} H^{2}}{L(1, \mathbb{X})^{2}}, X\right)\right)+O\left(\frac{H^{2}(\log \log H)^{3}}{\log H}\right)
\end{aligned}
$$


THE NUMBER OF IMAGINARY QUADRATIC FIELDS WITH A GIVEN CLASS NUMBER 5

Finally, using Proposition 1 of [1] (which states that the probability that $L(1, \mathbb{X}) \leq$ $\pi^{2} /\left(6 e^{\gamma} \tau\right)$ is $\exp \left(-e^{\tau-C_{1}} / \tau(1+o(1))\right.$ for some explicit constant $\left.C_{1}\right)$, we obtain

$$
\begin{aligned}
\mathbb{E}\left(\min \left(\frac{\pi^{2} H^{2}}{L(1, \mathbb{X})^{2}}, X\right)\right) & =\pi^{2} H^{2} \cdot \mathbb{E}\left(L(1, \mathbb{X})^{-2}\right)+O_{A}\left(\frac{H^{2}}{(\log H)^{A}}\right) \\
& =\frac{\pi^{2} \zeta(2)}{\zeta(3)} H^{2}+O_{A}\left(\frac{H^{2}}{(\log H)^{A}}\right) .
\end{aligned}
$$

Combining this estimate with equations (2.3),$(2.6)-(2.9)$, and noting that $\left(H e^{\lambda N}\right)^{2}-$ $H^{2} \ll H^{2}(\log \log H)^{3} / \log H$ completes the proof.

Proof of Theorem 1.2. Let $X, T$ and $c$ be as in the proof of Theorem 1.1. Let $\mathcal{D}(x)=$ $\{p \leq x: p \equiv 3(\bmod 4)\}$. Then similarly to (2.1), one has (see equation 3.6 of [2])

$$
\sum_{\substack{h \leq H \\ h \text { odd }}} \mathcal{F}(h)=\sum_{\substack{p \in \mathcal{D}(X) \\ h(-p) \leq H}} 1+O_{A}\left(\frac{H^{2}}{(\log H)^{A}}\right) .
$$

Let $\{\mathbb{Y}(p)\}$ be independent random variables taking the values 1 and -1 with equal probabilities $1 / 2$, and define

$$
L(1, \mathbb{Y})=\prod_{p}\left(1-\frac{\mathbb{Y}(p)}{p}\right)^{-1}
$$

To obtain (1.2), the authors of [2] prove that assuming GRH (see Theorem 3.3 of [2]) we have

$$
\sum_{p \in \mathcal{D}(x)} L\left(1, \chi_{p}\right)^{z}=|\mathcal{D}(x)| \cdot \mathbb{E}\left(L(1, \mathbb{Y})^{z}\right)+O_{\epsilon}\left(x^{1 / 2+\epsilon}\right),
$$

uniformly for all complex numbers $z$ such that $|z| \leq(\log x) /\left(500(\log \log x)^{2}\right)$, where $L\left(s, \chi_{p}\right)$ is the Dirichlet $L$-function attached to the Kronecker symbol $\chi_{p}=\left(\frac{-p}{\cdot}\right)$. Then, by partial summation together with Dirichlet's class number formula, they deduced that (see [2], p. 19)

$$
\sum_{p \in \mathcal{D}(X)} h(-p)^{-s}=\pi^{s} \cdot \mathbb{E}\left(L(1, \mathbb{Y})^{s}\right) \int_{1}^{X} x^{-s / 2} d|\mathcal{D}(x)|+O_{\epsilon}\left(X^{1 / 2+\epsilon}\right),
$$

for all complex numbers $s$ with $\operatorname{Re}(s)=c$ and $|s| \leq T$.

The proof of Theorem 1.2 then follows along the same lines of the proof of Theorem 1.1 by using (2.10) instead of (2.4).

\section{REFERENCES}

[1] A. Granville and K. Soundararajan, The distribution of values of $L\left(1, \chi_{d}\right)$. Geom. Funct. Anal. 13 (2003), no. 5, 992-1028.

[2] S. Holmin, N. Jones, P. Kurlberg, C. McLeman, K. L. Petersen, Missing class groups and class number statistics for imaginary quadratic fields. Preprint, 28 pages. arXiv:1510.04387. 
[3] K. Soundararajan, The number of imaginary quadratic fields with a given class number. Hardy-Ramanujan J. 30 (2007), 13-18.

[4] T. Tatuzawa, On a theorem of Siegel. Jap. J. Math. 21 (1951), 163-178.

Department of Mathematics and Statistics, York University, 4700 Keele Street, Toronto, ON, M3J1P3 CANADA

E-mail address: lamzouri@mathstat.yorku.ca 